and coordination may be more sensitive indicators of the adverse effects of lead on the central nervous system than tests of intelligence and academic achievement. Lead poisoning is now defined as a blood lead level greater than or equal to $10 \mathrm{mcg} / \mathrm{dL}$. Children aged 6 months to 6 years and those living in older housing are the highest priority for screening, mandatory in many States.

\title{
INTRACRANIAL TUMORS
}

\section{MENINGIOMAS: MRI AND PATHOLOGICAL FINDINGS}

MRIs and histopathological studies of five children, aged 3 months to 16 years, with extra-axial meningiomas are reported from the Arkansas Children's Hospital and University of Arkansas for Medical Sciences, Little Rock, AR. Two tumors were supratentorial and three were in the posterior fossa. Two located on the tentorium presented with headache, and one at the foramen magnum caused quadriparesis. All were hyperintense on proton density- and T2-weighted images and showed intense contrast enhancement on T1-weighted images. Two were meningotheliomatous, one transitional, one chordoid, and one hemangiopericytic variant of meningioma. Chromosome 22 deletions were found in two of four tumors studied. (Glasier CM et al. Meningiomas in children: MR and histopathologic findings. AINR Jan/Feb 1993; 14: 237-241). (Reprints: Charles M Glasier MD, Department of Radiology, Arkansas Children's Hospital, 800 Marshall Street, Little Rock, AR 72202).

COMMENT. Meningiomas in children are rare (1 to $3 \%$ of intracranial tumors) and much less common than in adults (15\%). About $25 \%$ of meningiomas in children are associated with neurofibromatosis, especially type 2 .

\section{HEADACHE AS FIRST SYMPTOM OF CEREBRAL TUMOR}

Five children, ranging in age from 10 months to 4 years, all presenting initially with headache, with or without vomiting, and in whom the final diagnosis was intracranial tumor, are reported from Petah Tiqva, Israel. The initial neurologic examination, including the fundi, was normal in three patients with craniopharyngioma, pinealoma, and cerebellar astrocytoma. Two patients with medulloblastoma and fourth ventricle choroid plexus papilloma had abnormal neurologic findings at presentation. The authors stress the need for neuroimaging studies in children younger than 4 years of age who have headache, especially if accompanied by vomiting, even if the neurologic exam is normal. (Straussberg R, Amir J. Headaches in children younger than 7 years: are they really benign? Arch Neurol Feb 1993; 0ㅜ 130 [Letter to the Editor] ). 